\title{
FLOaTing toward new standards in locally advanced resectable gastroesophageal cancer
}

\author{
Lorenzo Fornaro $^{1}$, Caterina Vivaldi ${ }^{1,2}$, Lorenzo Calvetti ${ }^{3}$, Alessandro Cappetta ${ }^{3}$, Alfredo Falcone ${ }^{1,4}$, \\ Giuseppe Aprile ${ }^{3}$
}

${ }^{1}$ Unit of Oncology 2, Azienda Ospedaliero-Universitaria Pisana, Pisa, Italy; ${ }^{2}$ Department of Clinical and Experimental Medicine, University of Pisa, Pisa, Italy; ${ }^{3}$ Department of Oncology, San Bortolo General Hospital, Vicenza, Italy; ${ }^{4}$ Department of Translational Research and New Technologies in Medicine, University of Pisa, Pisa, Italy

Correspondence to: Dr. Lorenzo Fornaro. Unit of Oncology 2, Azienda Ospedaliero-Universitaria Pisana, Via Roma 67, 56126 Pisa, Italy.

Email: lorenzo.fornaro@gmail.com.

Provenance: This is an invited article commissioned by the Academic Editor Weijun Jiang (Nanjing Normal University, Department of Reproductive and Genetics, Institute of Laboratory Medicine, Jinling Hospital, Nanjing University School of Medicine, Nanjing, China).

Comment on: Al-Batran SE, Homann N, Pauligk C, et al. Perioperative chemotherapy with fluorouracil plus leucovorin, oxaliplatin, and docetaxel versus fluorouracil or capecitabine plus cisplatin and epirubicin for locally advanced, resectable gastric or gastro-oesophageal junction adenocarcinoma (FLOT4): a randomised, phase 2/3 trial. Lancet 2019;393:1948-57.

Submitted Dec 15, 2019. Accepted for publication Dec 24, 2019.

doi: $10.21037 /$ jtd.2019.12.103

View this article at: http://dx.doi.org/10.21037/jtd.2019.12.103

\section{Introduction}

The optimal management of gastric adenocarcinoma (GC) and gastroesophageal junction adenocarcinoma (GEJC) remains a critical challenge for medical oncologists. Indeed, this is a relevant burden since GC still stands fifth among the most common tumors and is the third leading cause of cancer-related death worldwide (1).

Moreover, most patients with apparently localized disease at diagnosis experience recurrence after surgical resection (2). Moving from this evidence, several efforts have been conducted in order to improve patient prognosis by combining systemic chemotherapy (CT) in the algorithm of resectable GC and chemoradiotherapy (CRT) in resectable GEJC (3). The FLOT4 study is the most recently published trial addressing this issue and established a new therapeutic option for locally advanced GC and GEJC patients (4). Here we discuss the study design and results and put the data in context in light of the available literature evidence.

\section{Where did we stand before FLOT4?}

Multiple trials investigated the role of adjuvant chemotherapy after resection in GC and GEJC in Western countries, but results were disappointing $(3,4)$. In this dismal scenario, two randomized phase 3 trials established perioperative CT as standard of care. MAGIC and FNCLCC-FFCD studies compared fluoropyrimidinine plus platinum-based chemotherapy administered before and after surgery in stage I-III resectable GC and GEJC $(5,6)$. Despite some differences in the populations enrolled (a higher proportion of GEJC cases were enrolled in FNCLCC-FFCD), study regimens [epirubicin, cisplatin and 5-fluorouracil (ECF) in MAGIC vs. cisplatin and 5-fluorouracil (CF) in FNCLCCFFCD] and number of cycles administered before and after treatment (3 in MAGIC vs. 2-3 preoperatively and 3-4 postoperatively in FNCLCC-FFCD), UK and French investigators reached the same conclusions, demonstrating a $13 \%$ and $14 \%$ improvement, respectively, in 5 -year overall survival (OS) with CT compared with surgery alone (4-6). CT proved superior also in terms of other endpoints, such as progression-free survival (PFS) and tumor pathologic downstaging. Moreover, moving CT preoperatively significantly improved safety, with almost all patients completing the scheduled preoperative CT cycles: on the other hand, tolerance to adjuvant CT was confirmed suboptimal, as the percentage of patients completing postoperative CT dropped to less than $50 \%$. On the basis of these data, perioperative CT with CF (with or without 
epirubicin) was implemented in guidelines (7).

As described above, GEJC cases (Siewert I-III) were included in MAGIC and FNCLCC-FFCD studies, thus making perioperative CT a suitable option also for upper lesions. However, GEJC patients were also contemporarily enrolled in preoperative CRT trials, either alone or with esophageal tumors (4). Among the most relevant trials in this setting, CROSS compared weekly carboplatin plus paclitaxel concomitant with radiation and followed by surgery and surgery alone among patients with esophageal cancer or GEJC (both adenocarcinoma and squamous cell carcinoma were included): the study showed a $14 \%$ increase in 5-year OS with preoperative CRT (8). CRT proved to be safe and resulted in consistent tumor downstaging, with a pathologic complete response (pCR) rate of $23 \%$ among patients with adenocarcinoma. The parallel, smaller POET study focused on GEJC patients only, demonstrating a trend toward improved OS with the addition of radiation plus cisplatin and etoposide after induction CT with CF compared with CF alone: however, the study did not complete planned accrual and therefore conclusions suffer from limited power (9). Moreover, the authors reported a trend toward a non-significant increase of in-hospital mortality with CRT (10.2\% vs. $3.8 \%)$.

\section{Summary of FLOT4 study results}

Moving from the evidence of a significant, despite limited, survival advantage with docetaxel added to CF doublet in advanced disease (10) and considering the improved tolerability reported with the FLOT schedule (biweekly infusional 5-fluorouracil, oxaliplatin and docetaxel) (11), the German AIO group conducted a randomized phase 2-3 trial evaluating such a triplet schedule in non-metastatic GC and GEJC patients with stage cT2 or more or $\mathrm{cN}+$ disease (4). Four cycles of FLOT before and after surgery were compared with 3 cycles of ECF/ECX (epirubicin/ cisplatin/5-fluorouracil or capecitabine) in the control arm. In the first publication of the phase 2 part of the trial including 300 patients, FLOT significantly increased the rate of pCRs compared to the MAGIC regimen (16\% vs. $6 \%, \mathrm{P}=0.02$; pCRs plus subtotal responses: $37 \%$ vs. $23 \%$, $\mathrm{P}=0.02$ ) (12). As expected, pCRs were observed mainly in the subgroup with intestinal histology $(23 \%$ vs. $10 \%$ in the two arms), whereas remained sporadic in the diffuse subset (3\% in both arms).

Al-Batran and colleagues recently published the results of the phase 3 part of FLOT4, aiming at comparing FLOT and ECF/ECX in terms of OS (4). Overall 716 patients were randomized: main patient characteristics as well as study results are presented in Table 1. At a median follow up of 43 months among surviving patients, FLOT demonstrated a statistically and clinically meaningful OS improvement compared to anthracycline-based triplet (median: 50 vs. 35 months; 5-year OS: $45 \%$ vs. $36 \%$; HR 0.77, 95\% CI: 0.63-0.94). Moreover, FLOT also overcame ECF/ECX in secondary endpoints, such as disease-free survival (DFS) (median: 30 vs. 18 months) and rate of R0 resections (85\% vs. $78 \%$ ). At subgroup analysis, the superiority of FLOT was confirmed independently of age, presence of signet-ring cell histology, tumor location and clinical $\mathrm{T}$ or $\mathrm{N}$ stage.

With regards to safety, FLOT did not increase toxic deaths nor surgical morbidity and mortality and was not associated with higher rates of hospitalizations for toxicity. While severe (i.e., grade 3-4) nausea (16\% vs. 7\%) and vomiting ( $8 \%$ vs. $2 \%)$, anemia $(6 \%$ vs. $3 \%)$ and thromboembolic events ( $6 \%$ vs. $3 \%)$ were more frequent with ECF/ECX, neutropenia (51\% vs. 39\%; febrile neutropenia: $2 \%$ vs. $1 \%$ ), infections (18\% vs. $9 \%$ ), diarrhea (10\% vs. $4 \%$ ) and peripheral neuropathy (7\% vs. $2 \%)$ were more common with FLOT.

\section{How does FLOT4 fit in the algorithm?}

In light of the OS benefit reported in FLOT4, FLOT has been recently recognized as the new standard perioperative regimen in resectable GC and $\operatorname{GEJC}(3,13)$. Indeed, results are corroborated by high internal strength (i.e., superiority of FLOT over ECF/ECX in all endpoints) as well as external validity (i.e., results for the control arm are super imposable with those reported in MAGIC or FNCLCCFFCD). Safety concerns about intensive docetaxel-based triplets have been scaled down, demonstrating that such a schedule is feasible with adequate patient selection.

$\mathrm{CF}$, with or without epirubicin, represented the recommended choice before FLOT4 $(5,6)$. FLOT is a simplified, biweekly regimen, which allows a more convenient 5-fluorouracil infusion (24 hours, compared with the prolonged infusions used in previous perioperative CT trials) and makes oxaliplatin, which has been proved as less toxic than cisplatin in meta-analyses (14), enter the neoadjuvant scenario.

FLOT4 protocol also scheduled the most accurate staging procedures among the perioperative CT trials, thus making the observed data robust and trustworthy (4). All patients had endoscopy, endoscopic ultrasound and 
Table 1 FLOT4: summary of main results

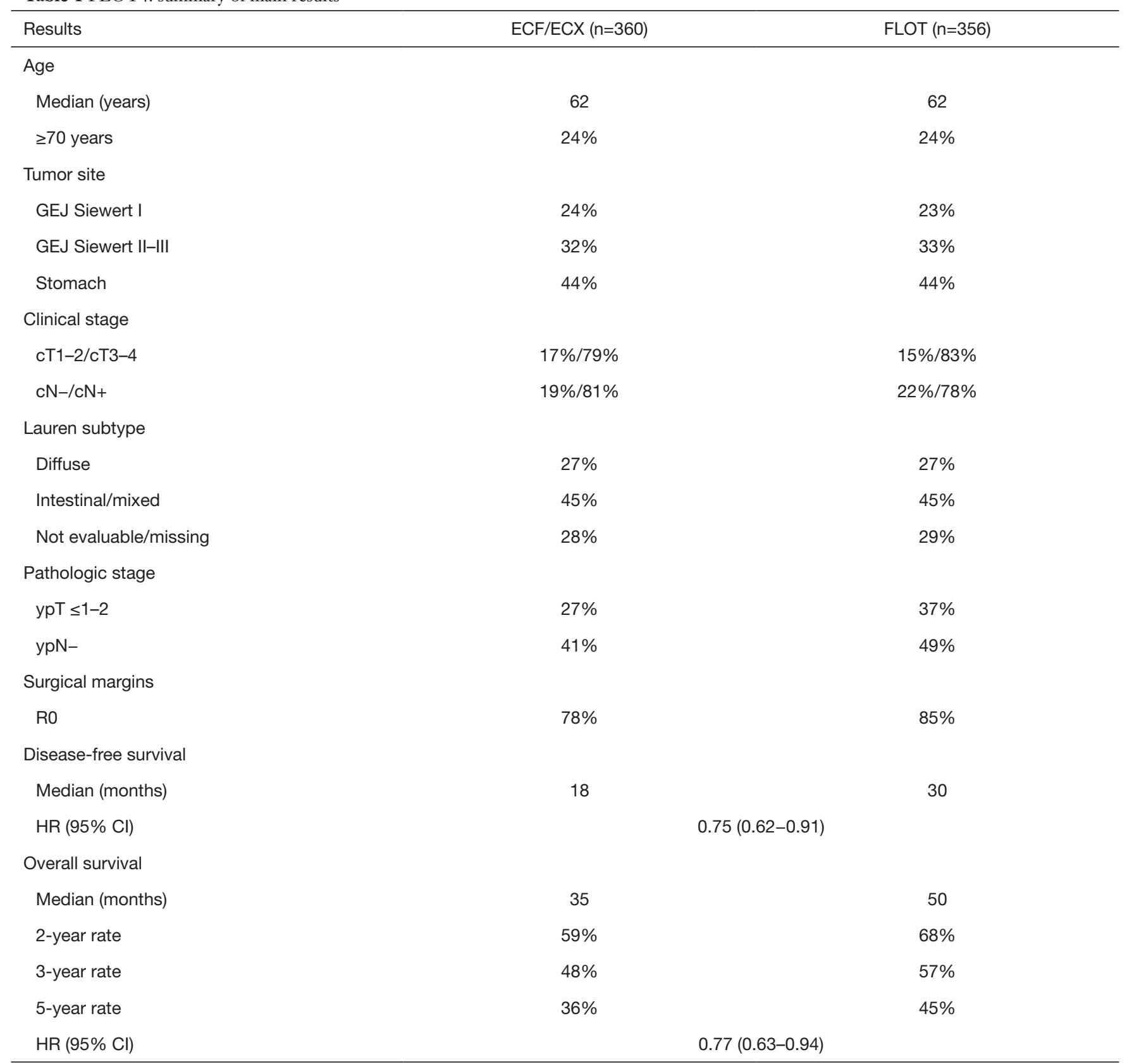

ECF, epirubicin, cisplatin and 5-fluorouracil; ECX, epirubicin cisplatin capecitabine; GEJ, gastroesophageal junction; HR (95\% Cl), hazard ratio (95\% confidence interval); $n$, number (of patients).

computed tomography or magnetic resonance imaging, while laparoscopy was recommended but not mandatory. Therefore, FLOT4 protocol could be useful also to establish a reference staging work-up for everyday multidisciplinary assessment of GC and GEJC cases.

In light of the results reported in FLOT4, there is no doubt that perioperative FLOT represents the best alternative for fit patients in GC. But is this conclusion still valid for GEJC, particularly Siewert I disease? For proximal location, neoadjuvant CRT can be considered too, as it provided OS benefit over surgery alone in randomized trials in esophageal cancer and $\operatorname{GEJC}(2,3)$. Whether radiation is combined with carboplatin plus paclitaxel or with CF, preoperative CRT results in significant pCR 
rates, higher R0-resection rates and longer OS compared to upfront surgical resection $(2,3)$. Obviously, we should be cautious in deriving definitive conclusions from crosstrial comparisons. With this limitation in mind, we should consider that perioperative FLOT overcame the results of an active multimodality treatment in the control arm, whereas neoadjuvant CRT has not yet demonstrated a clear OS advantage over CT in GEJC (9). Moreover, CRT is often considered superior to CT in terms of pathologic responses (3). Looking at the data available for CRT more in details, this assumption is clearly convincing in squamous cell carcinoma of the esophagus $(2,3,8)$ : however, when compared with pathologic findings in FLOT4 $(4,12)$, results with CRT in esophageal and GEJC seem not to be unequivocally greater. In addition, subgroup analyses in CROSS found lower OS benefit for CRT in the adenocarcinoma subset (8). On the contrary, relative risk reduction for mortality in FLOT4 was the same in GC and GEJC (4). Finally, CT and CRT differ in terms of toxicities, and the different safety profiles of the two strategies should be considered in the personalized approach to GEJC cases $(2-4,8,9)$.

Another issue to be discussed is represented by the optimal management of patients affected by disease staged as cT2N0. These patients were eligible for FLOT4, however the exact number is not reported (4). Subgroup OS analyses revealed a greater benefit for cT1/2 (compared to cT3/4) and $\mathrm{cN}-$ (compared to $\mathrm{cN}+$ ) tumors (4). However, it is difficult to assess the impact of perioperative CT in this patient subset and multidisciplinary discussion should take into account other patient-related (e.g., age, performance status, motivation, comorbidities) and tumor-related [such as microsatellite instability (MSI) status] (15) parameters.

\section{How can we move forward?}

FLOT4 confirmed previous data from MAGIC and FNCLCC-FFCD about the suboptimal compliance to postoperative CT, with less than $50 \%$ of the patients completing all planned cycles (4-6). As completion of CT might be a key factor to maximize impact of systemic treatment on OS (16), supportive measures should be planned and offered to all patients before and during treatment, such as nutritional counseling and strict monitoring of hematologic toxicities with eventual G-CSF support (received by $34 \%$ of the patients in the FLOT arm) (4). Preliminary reports of primary G-CSF prophylaxis indicate that such an approach might reduce the risk of severe neutropenia, but impact on dose intensity and long-term outcome remains to be clarified (17). Moving all CT cycles before surgery could be another interesting option in order to improve safety and treatment exposure: however this could be counterbalanced by higher risk of toxicity and might potentially delay surgery in some cases (4). Moreover, considering the lack of predictive biomarkers (beyond MSI status (15), discussed in the next paragraphs) the risk of over treating patients with useless CT cycles postoperatively is still to be considered: indeed, MAGIC authors found out that patients with nodepositive disease at pathology assessment after preoperative CT had the worse outcome, thus questioning the utility of postoperative therapy in such cases (18).

Three randomized trials are now either comparing perioperative FLOT with neoadjuvant CRT according to CROSS schedule (ESOPEC and Neo-AEGIS) $(19,20)$ in esophageal cancer and GEJC or trying to integrate preoperative CRT with perioperative CT (TOPGEAR) (21) in GC and GEJC (Table 2). Results of these studies will answer the questions about the optimal approach to GEJC as well as the role of neoadjuvant CRT in the setting of a perioperative CT strategy $(2,3)$. Preliminary data for TOPGEAR confirmed that CRT can be combined preoperatively with $\mathrm{CT}$, with apparent no increase in toxicity or surgical morbidity (21).

The combination of FLOT with biologic agents [mainly anti-HER2, anti-angiogenics, or immune checkpoint inhibitors (ICIs)] is currently under extensive investigation (3) (Table 2). Unfortunately, with the exception of trastuzumab (with or without pertuzumab) among HER2-positive cases, no molecular selection is applied. In light of the recent results in the advanced setting (22), this could be critical particularly for the development of ICIs in resectable disease.

Notably, a recent meta-analysis pooled the evidence about the role of MSI status in resectable GC or GEJC (15). In summary the authors analyzed single-patient data from four randomized trials (MAGIC, ITACA-S, ARTIST, CLASSIC) and convincingly confirmed the positive prognostic role of MSI-high status (reported in 10-20\% of the cases in the adjuvant setting), as it is associated with longer DFS (HR 1.88, 95\% CI: 1.28-2.76) and OS (HR 1.78, 95\% CI: 1.17-2.73). Of interest, among MSIhigh cases, no significant benefit of CT or CRT could be proved for DFS (HR 1.27, 95\% CI: 0.53-3.04) and OS (HR $1.50,95 \%$ CI: $0.55-4.12)$, and an apparent trend toward a detrimental effect of treatment over surgery alone was indeed reported. This was also suggested in the metastatic setting (23). With the limitations of a meta-analysis 
Table 2 Main ongoing randomized phase 2-3 trials in resectable GC and GEJC

\begin{tabular}{|c|c|c|c|c|c|}
\hline Study & $\mathrm{n}$ & Objective & Patients & Selection criteria & ClinicalTrials.gov ID \\
\hline TOPGEAR & 752 & OS & GC and GEJC & $\geq \mathrm{cT} 3$ or $\mathrm{cN}+$ & NCT01924819 \\
\hline ESOPEC & 438 & OS & EC and GEJC & $\geq \mathrm{cT} 2$ or $\mathrm{cN}+$ & NCT02509286 \\
\hline Neo-AEGIS & 366 & os & EC and GEJC & cT2-3, N0-3 & NCT01726452 \\
\hline \multicolumn{6}{|c|}{ Anti-HER2 antibodies } \\
\hline PETRARCA & 404 & $\begin{array}{c}\text { pCR (ph. 2), PFS } \\
\text { (ph. 3) }\end{array}$ & GC and GEJC (HER2+) & $\geq \mathrm{cT} 2$ or $\mathrm{cN}+$ & NCT02581462 \\
\hline INNOVATION & 225 & $\mathrm{pCR}$ & GC and GEJC (HER2+) & Clinical stage Ib-III & NCT02205047 \\
\hline RTOG-1010 & 591 & DFS & EC and GEJC (HER2+) & cT1 $1 \mathrm{~N}+$ or cT2-3, any $\mathrm{N}$ & NCT01196390 \\
\hline \multicolumn{6}{|c|}{ Immune check-point inhibitors } \\
\hline DANTE & 295 & PFS & GC and GEJC & $\geq \mathrm{cT} 2$ or $\mathrm{cN}+$ & NCT03421288 \\
\hline KEYNOTE-585 & 860 & $\begin{array}{l}\text { OS, EFS, pCR } \\
\text { and AEs }\end{array}$ & GC and GEJC & $\geq \mathrm{cT} 3$ or $\mathrm{cN}+$ & NCT03221426 \\
\hline VESTIGE & 240 & DFS & Lower EC, GEJC and GC & ypN1-3 or ypN0, R1 & NCT03443856 \\
\hline ONO-4538-38 & 700 & RFS & GOJC and GC & Pathologic stage III, RO & NCT03006705 \\
\hline
\end{tabular}

AEs, adverse events; DFS, disease-free survival; EC, esophageal adenocarcinoma; EFS, event-free survival; GC, gastric adenocarcinoma; GEJC, gastroesophageal junction adenocarcinoma; n, number (of patients); OS, overall survival; pCR, pathologic complete response; PFS, progression-free survival.

including heterogeneous trials in terms of populations, ethnicity and treatments provided, these data could be useful to inform practice when the benefit of perioperative $\mathrm{CT}$ is questionable (e.g., cT2N0 cases). However, none of the trials included in the meta-analysis tested FLOT (or a docetaxel-based triplet) as perioperative therapy: therefore, one could argue if the results are still the same when FLOT is administered. Hence, translational analyses of the FLOT4 dataset will be of paramount relevance to answer questions about MSI and other potential biomarkers.

\section{Conclusions}

FLOT4 will guide clinical practice in resectable GC and GEJC from now on: if this study does not change the preferred strategy (perioperative CT) in this setting, it establishes a new standard regimen for patients in good performance status, able to tolerate triplet CT. When discussing these cases in the multidisciplinary team, all clinicians should consider the result of FLOT4 and, if applicable, evaluate the feasibility of FLOT in each single case. In Siewert I tumors, too, the benefit of FLOT and its safety profile should be compared with the alternative CRT schedules: in our opinion, FLOT may be preferable even in these situations, particularly for its potentially greater impact on micrometastatic disease and similar pCR rates compared to CRT. Phase 3 trials are ongoing in order to definitively compare CT and CRT in this population.

MSI status assessment could be useful for a more informed discussion with patients about prognosis, but uncertainties about its role as predictive biomarker should be acknowledged. Future research will hopefully add information about the role of MSI status in GC and GEJC. Finally, FLOT is now viewed as the CT backbone to be implemented in the assessment of biologic agents: safety of intensive CT triplet with new drugs should be carefully 
evaluated, and deep translational investigation should be run in parallel with clinical activities to move beyond TNM stage and finally tailor the approach in specific molecular patient subgroups.

\section{Acknowledgments}

None.

\section{Footnote}

Conflicts of Interest: The authors have no conflicts of interest to declare.

Ethical Statement: The authors are accountable for all aspects of the work in ensuring that questions related to the accuracy or integrity of any part of the work are appropriately investigated and resolved.

\section{References}

1. Siegel RL, Miller KD, Jemal A. Cancer statistics, 2019. CA Cancer J Clin 2019;69:7-34.

2. Wagner AD, Lordick F, Grabsch HI, et al. Multidisciplinary management of stage II-III gastric and gastro-oesophageal junction cancer. Eur J Cancer 2020;124:67-76.

3. Fornaro L, Vasile E, Aprile G, et al. Locally advanced gastro-oesophageal cancer: Recent therapeutic advances and research directions. Cancer Treat Rev 2018;69:90-100.

4. Al-Batran SE, Homann N, Pauligk C, et al. Perioperative chemotherapy with fluorouracil plus leucovorin, oxaliplatin, and docetaxel versus fluorouracil or capecitabine plus cisplatin and epirubicin for locally advanced, resectable gastric or gastro-oesophageal junction adenocarcinoma (FLOT4): a randomised, phase 2/3 trial. Lancet 2019;393:1948-57.

5. Cunningham D, Allum WH, Stenning SP, et al. Perioperative chemotherapy versus surgery alone for resectable gastroesophageal cancer. $\mathrm{N}$ Engl J Med 2006;355:11-20.

6. Ychou M, Boige V, Pignon JP, et al. Perioperative chemotherapy compared with surgery alone for resectable gastroesophageal adenocarcinoma: An FNCLCC and FFCD multicenter phase III trial. J Clin Oncol 2011;29:1715-21.

7. Smyth EC, Verheij M, Allum W, et al.; ESMO Guidelines Committee. Gastric cancer: ESMO Clinical Practice
Guidelines for diagnosis, treatment and follow-up. Ann Oncol 2016;27:v38-49.

8. van Hagen P, Hulshof MC, van Lanschot JJ, Steyerberg EW, van Berge Henegouwen MI, Wijnhoven BP, et al. Preoperative chemoradiotherapy for esophageal or junctional cancer. N Engl J Med 2012;366:2074-84.

9. Stahl M, Walz MK, Stuschke M, et al. Phase III comparison of preoperative chemotherapy compared with chemoradiotherapy in patients with locally advanced adenocarcinoma of the esophagogastric junction. J Clin Oncol 2009;27:851-6.

10. Van Cutsem E, Moiseyenko VM, Tjulandin S, et al. Phase III study of docetaxel and cisplatin plus fluorouracil compared with cisplatin and fluorouracil as first-line therapy for advanced gastric cancer: a report of the V325 Study Group. J Clin Oncol 2006;24:4991-7.

11. Al-Batran SE, Hartmann JT, Hofheinz R, et al. Biweekly fluorouracil, leucovorin, oxaliplatin, and docetaxel (FLOT) for patients with metastatic adenocarcinoma of the stomach or esophagogastric junction: a phase II trial of the Arbeitsgemeinschaft Internistische Onkologie. Ann Oncol 2008;19:1882-7.

12. Al-Batran SE, Hofheinz RD, Pauligk C, et al. Histopathological regression after neoadjuvant docetaxel, oxaliplatin, fluorouracil, and leucovorin versus epirubicin, cisplatin, and fluorouracil or capecitabine in patients with resectable gastric or gastro-oesophageal junction adenocarcinoma (FLOT4-AIO): results from the phase 2 part of a multicentre, open-label, randomised phase $2 / 3$ trial. Lancet Oncol 2016;17:1697-708.

13. Pentheroudakis G. Recent eUpdates to the ESMO Clinical Practice Guidelines on Hepatocellular Carcinoma, Cancer of the Pancreas, Soft Tissue and Visceral Sarcomas, Cancer of the Prostate and Gastric Cancer. Ann Oncol 2019;30:1395-7.

14. Ter Veer E, Haj Mohammad N, van Valkenhoef G, et al. The efficacy and safety of first-line chemotherapy in advanced esophagogastric cancer: a network meta-analysis. J Natl Cancer Inst 2016;108. doi: 10.1093/jnci/djw166.

15. Pietrantonio F, Miceli R, Raimondi A, et al. Individual patient data meta-analysis of the value of microsatellite instability as a biomarker in gastric cancer. J Clin Oncol 2019;37:3392-400.

16. Karagkounis G, Squires MH 3rd, Melis M, et al. Predictors and prognostic implications of perioperative chemotherapy completion in gastric cancer. J Gastrointest Surg 2017;21:1984-92.

17. Crampton KM, Wood J, Wong H, et al. Evaluation of the 
introduction of primary G-CSF prophylaxis to the FLOT chemotherapy regimen. Ann Oncol 2019;30:v300.

18. Smyth EC, Fassan M, Cunningham D, et al. Effect of pathologic tumor response and nodal status on survival in the Medical Research Council Adjuvant Gastric Infusional Chemotherapy trial. J Clin Oncol 2016;34:2721-7.

19. Hoeppner J, Lordick F, Brunner T, et al. ESOPEC: prospective randomized controlled multicenter phase III trial comparing perioperative chemotherapy (FLOT protocol) to neoadjuvant chemoradiation (CROSS protocol) in patients with adenocarcinoma of the esophagus (NCT02509286). BMC Cancer 2016;16:503.

20. Reynolds JV, Preston SR, O'Neill B, et al. ICORG 10-14: NEOadjuvant trial in Adenocarcinoma of the oEsophagus and oesophagoGastricjunction International Study (NeoAEGIS). BMC Cancer 2017;17:401.

Cite this article as: Fornaro L, Vivaldi C, Calvetti L, Cappetta A, Falcone A, Aprile G. FLOaTing toward new standards in locally advanced resectable gastroesophageal cancer. J Thorac Dis 2019;11(12):5694-5700. doi: 10.21037/jtd.2019.12.103
21. Leong T, Smithers BM, Haustermans K, et al. TOPGEAR: A randomized, phase III trial of perioperative ECF chemotherapy with or without preoperative chemoradiation for resectable gastric cancer: interim results from an international, intergroup trial of the AGITG, TROG, EORTC and CCTG. Ann Surg Oncol 2017;24:2252-8.

22. Shitara K, Van Cutsem E, Bang YJ, et al. Pembrolizumab with or without chemotherapy vs chemotherapy in patients with advanced G/GEJ cancer (GC) including outcomes according to microsatellite instability-high (MSI-H) status in KEYNOTE-062. Ann Oncol 2019;30:v878-9.

23. Janjigian YY, Sanchez-Vega F, Jonsson P, et al. Genetic predictors of response to systemic therapy in esophagogastric cancer. Cancer Discov 2018;8:49-58. 